\section{Prevalência e fatores de risco \\ de anemia em mães e filhos no \\ Estado de Pernambuco}

\section{Prevalence and risk factors of anemia among women and their children in the State of Pernambuco}

Shirley Cristina Lima e Silva ${ }^{1}$

Malaquias Batista Filho

Teresa Cristina Miglioli ${ }^{3}$

${ }^{1}$ Faculdade do Vale do Ipojuca - FAVIP

${ }^{2}$ Instituto Materno Infantil Profo Fernando Figueira (IMIP)

${ }^{3}$ Centro de Pesquisa Aggeu Magalhães - CPqAM/Fiocruz

\section{Resumo}

Objetivo: Estudar a prevalência e fatores de risco de anemia em mães e filhos menores de 5 anos, no Estado de Pernambuco, em três espaços geográficos: Região Metropolitana do Recife (RMR); Interior Urbano (IU) e Interior Rural (IR). Método: Estudo seccional, em amostra representativa de 807 mulheres adultas e 827 crianças menores de 5 anos, sorteadas em 3 estágios (municípios, setores censitários e famílias), possibilitando 523 pareamentos mães x filhos biológicos. A hemoglobina foi determinada em aparelho portátil (HemoCue). Fez-se avaliação de fatores de risco (biológicos, socioeconômicos, ambientais e acesso a serviços de saúde). Resultados: No conjunto dos resultados, $21,8 \%$ das mulheres e $46,9 \%$ das crianças tinham anemia, prevalências semelhantes no pareamento mães/filhos biológicos. As prevalências mais baixas de anemia foram encontradas no IU, enquanto as mais elevadas ocorreram no IR. Ocorreu associação entre a situação das mães e dos filhos, com um RP = 1,45, IC (95): 1,21-1,74. Quatro fatores de risco foram comuns às mães e filhos: tipo de ocupação das moradias, coleta de lixo, assistência pré-natal e distância do serviço de saúde. As crianças incorporam mais sete fatores: idade da mãe, idade da criança, espaço geográfico, baixa escolaridade das mães, baixa renda familiar, tamanho da família e falta de esgotos sanitários. Conclusão: A prevalência de anemia se diferencia por espaços geográficos, idade das crianças e condição de exposição de mães e filhos a fatores biológicos, sócioeconômicos e ambientais.

Palavras-chave: Anemia. Prevalência. Mães e filhos. Estado de Pernambuco. 


\section{Abstract}

Objective: To determine the prevalence and risk factors for anemia in mothers and their children under five years of age in the state of Pernambuco (Brazil) in three geographic areas: Metropolitan Recife (MR); Urban Interior (UI) and Rural Interior (IR). Method: A cross-sectional study was carried out with a representative sample of 807 adult women and 827 children under five years of age selected by lots in three stages (municipal, census sectors and families), which provided 523 biological mother/child pairings. Hemoglobin was determined by a portable hemoglobinometer (HemoCue). Risk factors (biological, socioeconomic, environmental and access to health services) were assessed. Results: A total of $21.8 \%$ of the women and $46.9 \%$ of the children had anemia, with a similar prevalence in the mother/child pairings. The lowest prevalence was found in the UI, while the highest rates occurred in the RI. There was an association between the situation of the mothers and children $(\mathrm{PR}=1.45$; 95\%CI: 1.21-1.74). Four risk factors were common to both mothers and children: type of residence, garbage collection, antenatal care, and distance from healthcare services. Children incorporated another seven factors: mother's age, child's age, geographic area, mother's low schooling, low family income, family size, and lack of sewage. Conclusion: The prevalence of anemia is different according to geographic space, child's age and exposure of mothers and children to biological, socioeconomic and environmental factors.

Keywords: Anemia. Prevalence. Mothers and Children. Pernambuco - Brazil

\section{Introdução}

Em termos de magnitude, as anemias configuram a principal endemia carencial do mundo, ocorrendo em cerca de 2.150 milhões de pessoas e se difundindo entre todos os continentes, grupos sociais e segmentos biológicos, embora predominando amplamente nas regiões pobres, nas famílias de baixa renda, no grupo de mulheres no período reprodutivo e em crianças menores de cinco anos ${ }^{1}$. Avaliações mais recentes chegam a dimensionar valores bem mais elevados, a exemplo do Comitê UNICEF/WHO, 1999, calculando que o problema poderia se estender a um contingente de 3,5 bilhões de pessoas, o que representaria uma prevalência global de aproximadamente $58 \%$ da população humana ${ }^{2}$. Deve-se esclarecer que, embora como problema de patologia humana, as anemias possam compreender o desfecho isolado ou combinado de variados fatores, desde os de natureza genética até múltiplas condições ambientais, na prática o que predomina em nível populacional é a gênese carencial, envolvendo sobretudo a deficiência quali ou quantitativa de ferro alimentar ${ }^{3}$. É uma condição tão comum que, sempre que a magnitude das anemias se torna elevada, passa a ser assumida como sinonímia de anemia carencial e até especificamente como ferropriva, inclusive com o reconhecimento dos comitês internacionais de especialistas encarregados de estabelecer conceito, normas e recomendações sobre o tema ${ }^{3}$.

No caso do Brasil, as anemias apresentam uma característica epidemiológica singular, por sua tendência de endemia progressiva documentada em alguns estudos de observações temporais em crianças de São Paulo, no Estado da Paraíba e em escolares do Recife, com aumentos de prevalência variando de $88 \%$ a $120 \%$, em intervalos compreendidos entre 10 a $21 \operatorname{anos}^{4-6}$. É um comportamento que contraria as tendências verificadas em outros problemas carenciais, como a redução da incidência do baixo peso ao nascer (em cerca de $40 \%$ 
nas regiões Norte e Nordeste nos últimos 25 anos), o declínio da desnutrição (déficit altura/idade) em aproximadamente $70 \%$, o desaparecimento da deficiência calórica em escala de saúde coletiva em populações adultas, o controle epidemiológico da carência de iodo e a provável tendência de desaparecimento da hipovitaminose $\mathrm{A}^{7}$.

Apesar da sua importância, dos custos relativamente baixos de sua avaliação em inquéritos populacionais e, ainda, da relativa simplicidade operacional das técnicas de diagnóstico tipo "screening", apenas três Estados (Pernambuco, Piauí e Sergipe) realizaram inquéritos laboratoriais em amostras representativas de sua população de crianças ${ }^{8-10}$, sendo que, no caso de Pernambuco e Piauí, a avaliação se estendeu ao segmento de mulheres com história reprodutiva. Nestas circunstâncias, a oportunidade de um estudo que, simultaneamente, possa representar os segmentos biológicos mais vulneráveis (mulheres no período reprodutivo e crianças menores de cinco anos), cobrindo o território de um Estado e incluindo variáveis socioeconômicas, ambientais e acesso aos serviços de saúde, configura uma iniciativa que pode oferecer informações interessantes para o dimensionamento, compreensão e novos estudos do problema. Objetiva-se, neste artigo, descrever a prevalência das anemias, sua distribuição no espaço geográfico e relação com possíveis fatores associados à sua ocorrência no binômio mães/filhos, na segunda metade da década de 90 (1997), no Estado de Pernambuco.

\section{Materiais e Métodos}

$\mathrm{O}$ artigo aqui apresentado utiliza-se do banco de dados da pesquisa "Saúde, nutrição, alimentação e condições socioeconômicas no Estado de Pernambuco" ${ }^{\prime \prime}$, derivando da criação de um arquivo secundário (ad hoc) com o resgate seletivo de informações sobre o nível de hemoglobina das crianças menores de 5 anos e mulheres com história reprodutiva, incluindo as mães da grande maioria das crianças investigadas.

\section{Amostragem}

Os procedimentos utilizados para a coleta de dados de campo da pesquisa básica, compreendendo um inquérito de 1.431 famílias, totalizando 7.748 pessoas distribuídas na Região Metropolitana do Recife (RMR), Interior Urbano (IU) e Interior Rural (IR), acham-se detalhadamente descritos no relatório Alimentação, Nutrição e Saúde no Estado de Pernambuco, organizado por Batista Filho e Romani $(2002)^{8}$, já desdobrado em outra publicação ${ }^{11}$. No referido estudo básico, considerando o indicador de maior interesse do inquérito (altura/idade $<2$ escores Z do padrão NCHS) ${ }^{12}$, admitindo um erro de $\pm 3 \%$, um nível de confiança de $95 \%$ e uma prevalência estimada de $30 \%$ (década anterior para o Nordeste), a amostra calculada foi de 2.012 crianças, para representar o universo de 849.500 menores de 5 anos. Para o caso das anemias em mães, com prevalência estimada em $25 \%$, erro de $\pm 3 \%$ e nível de confiança de $95 \%$, a amostra representativa para o Estado seria de 800 observações. Para as crianças, utilizando uma prevalência de $45 \%$ erro de \pm 4 e nível de confiança de $95 \%$, o tamanho da amostra resultava em 594 unidades de observação. No entanto, levando em conta o objetivo do pareamento mãe/filho biológico, adotou-se um "n" amostral mínimo também de 800 observações. Estes cálculos são representativos para o conjunto do Estado de Pernambuco, não se aplicando os mesmos critérios de dimensionamento para os três estratos geográficos (RMR, IU, e IR), de modo que os resultados destas subáreas devem ser usados, estatisticamente, para comparações entre si.

O processo de amostragem foi desenvolvido em três estágios. No primeiro, foram sorteados os municípios, com uma probabilidade proporcional à sua população; no segundo, foram selecionados aleatoriamente o(s) setor(es) censitário(s) dentro de cada município; finalmente, no terceiro estágio foram indicadas as famílias com crianças menores de 5 anos (crianças-índice) que seriam objeto do estudo. A escolha das fa- 
mílias e crianças foi feita por sorteio de uma residência inicial, a partir da qual, em sentido anti-horário da localização cartográfica do setor, seriam identificadas as unidades de observação: crianças e mulheres com história reprodutiva. Em cada setor seriam avaliadas em média $46 \pm 3$ crianças e mulheres com antecedentes de gravidez, entre as quais, necessariamente (se possível) as mães biológicas das crianças da amostra. Foram assim pesquisados 45 setores censitários (16 na RMR, 17 no IU e 12 no IR). No procedimento operacional, para cada três crianças e três mães, uma era indicada para compor a amostra prevista para os exames bioquímicos (hemoglobina, e retinol sérico para as crianças, mais as dosagens de glicose, colesterol e triglicerídios para as mães). Foi estabelecida uma margem adicional de $10 \%$ para compensar possíveis perdas nas avaliações de consistência e integralidade das informações. Antes da coleta de dados, as famílias sorteadas foram visitadas por um profissional de comunicação social, oportunizando o esclarecimento dos objetivos e procedimentos do estudo, sorteio e aceitação das pessoas que seriam examinadas.

O desenvolvimento do trabalho incluiu a confecção de 9 blocos de questionários, teste e adaptação dos protótipos propostos, elaboração de guias instrutivos, estabelecimento e teste do plano logístico para o trabalho coordenado de quatro sub-grupos de pesquisadores de campo (entrevistadores, avaliação do consumo alimentar, antropometria e coleta de amostras de sangue para determinações bioquímicas), mobilizando uma equipe de 22 pessoas (médicos, nutricionistas, sociólogos e técnicos de laboratório) durante quatro meses (janeiro a maio de 1997).

\section{Variáveis estudadas}

A determinação laboratorial de hemoglobina foi efetuada em aparelho portátil HemoCue com leitura direta e imediata, utilizando-se amostras de sangue coletado por venopunção ou, eventualmente, amostras obtidas da polpa digital.
Para discriminação da anemia, como variável dependente, foram adotados os pontos de corte recomendados pelo Comitê UNICEF/WHO'2: hemoglobina abaixo de $12 \mathrm{~g} / \mathrm{dL}$ para as mães e abaixo de $11 \mathrm{~g} / \mathrm{dL}$ para as crianças menores de 5 anos.

Como variáveis independentes foram consideradas:

Variáveis biológicas: idade das crianças (em meses) e das mães (em anos), e peso ao nascer das crianças, ordenado em três categoriais: baixo peso $(<2500 \mathrm{~g})$; peso insuficiente $(\geq 2.500<3.000 \mathrm{~g})$; peso normal $(\geq$ $3.000 \mathrm{~g})$. Os dados de peso ao nascer foram obtidos do cartão da criança.

Variáveis do macro e micro-ambiente: espaço geográfico Região Metropolitana do Recife (RMR), Interior Urbano (IU) e Interior Rural (IR), esgotamento sanitário (categorizado em rede geral e sem rede geral), destino do lixo (coletado e não coletado).

Condições socioeconômicas: escolaridade materna (anos de escolaridade), renda familiar total e renda familiar per capita (em salários mínimos), número de pessoas por domicílio ( $<5$ pessoas, $5-9$ pessoas e $\geq 10$ pessoas), regime de ocupação da residência (própria, alugada, cedida ou invadida).

Acesso aos serviços e ações de saúde: visita regular do Agente Comunitário de Saúde (ACS), distância (em km) do serviço de saúde mais próximo $(<1 \mathrm{~km} ; 1-3 \mathrm{~km} ; \geq 3$ km), assistência pré-natal da mãe (não, sim, número de consultas).

A associação das variáveis foi testada mediante a estimativa de "razão de prevalência de Mantel Haenzel” entre os grupos comparados, com um intervalo de confiança (IC) de 95\%. Em uma situação específica (comparação de prevalência de anemia entre mães e filhos por grupos etários das crianças), utilizou-se o teste de proporções percentuais para discriminar, estatisticamente, as possíveis diferenças nos resultados. Nas comparações para identificar fatores de risco, as análises estatísticas foram efetuadas mediante situações dicotômicas, considerando-se o grupo de menor ocorrência como referencial em relação ao de maior prevalência, nos casos em que a 
variável independente apresentava três ou mais alternativas, como se exemplifica com a estratificação de renda e de escolaridade. Para decisões estatísticas, admitiu-se um erro alfa de 0,05 .

Para a realização das análises epidemiológicas, foi utilizado o software "EPI-INFO 6”, versão 6.04, mediante a aplicação do teste de Mantel Haenzel, (razão de prevalência).

A pesquisa foi autorizada pelo Comitê de Ética do Centro de Ciências da Saúde da Universidade Federal de Pernambuco.

Os autores, através de documentação assinada e enviada a RBE, declaram não existir nenhum tipo de conflito de interesses.

\section{Resultados}

Na Tabela 1, acham-se descritas as características amostrais dos grupos estudados nos diversos espaços geográficos. Destaca-se, no conjunto de informações, o baixo nível educacional das mães $(40,1 \%$ com 4 anos ou menos de estudo) e de renda, principalmente nas famílias rurais ( $50 \%$ ganhando até $1 / 4$ de salário mínimo per capita) e a baixa cobertura em relação ao acesso à rede geral de esgotos sanitários: pouco mais de $1 / 4$ das residências.

Para o conjunto amostral de todo o Estado, a freqüência de anemia entre as mães foi de $21,8 \%$, enquanto nas crianças elevou-se para $46,9 \%$, apresentando, assim, uma razão de prevalência de 2,15. Entre as mães, os valores mais baixos $(15,9 \%)$ foram encontrados no IU, enquanto os mais elevados foram assinalados no IR $(24,7 \%)$, praticamente equivalentes aos obtidos na RMR (21,8\%). Em relação às crianças, a freqüência mais alta de anemia ocorreu na amostra do IR (56,6\%), em contraste com a prevalência mais baixa $(41,2 \%)$ detectada no interior urbano.

Como se verifica no segundo segmento (parte B da Tabela 2) os resultados referentes ao pareamento mãe/filho biológico não diferem da distribuição percentual encontrada no total da amostra do Estado. Provas estatísticas realizadas para compa- rar diferenças de prevalência entre mães e crianças, sejam ou não filhos biológicos, demonstraram que em todas as situações, com exceção da área urbana do interior (IU), a ocorrência de anemia foi mais de duas vezes maior entre as crianças (Tabela 2).

Ao analisar, caso a caso, a associação entre a ocorrência ou não de anemia na mãe com a ocorrência ou não de anemia em seu filho biológico, verificou-se que as duas condições se encontram relacionadas de forma estatisticamente significante. Assim, para o conjunto do Estado, as mães anêmicas apresentaram uma probabilidade de $66,3 \%$ de ocorrência de anemia em seus filhos, enquanto nas mães não anêmicas a chance de filhos também anêmicos baixou para $45,7 \%$. No interior rural, filhos de mães anêmicas apresentaram um risco de $74,3 \%$ de serem, também, portadores de anemia. Apenas no IU esta associação não se confirmou, sob o ponto de vista estatístico (Tabela 3).

Uma outra análise considerou a idade ( $<3$ anos / $\geq 3$ anos) como possível fator de diferenciação dos resultados de distribuição das anemias em crianças. Com este tratamento, a ocorrência do problema no grupo de menores de 36 meses foi de $57,6 \%$, enquanto nas crianças maiores de 36 meses situou-se em $24,6 \%$. A freqüência de anemia nas crianças com 36 meses ou mais, tornouse estatisticamente semelhante à verificada nas mães $(21,8 \%)$, segundo o teste de diferenças percentuais. Na comparação entre a prevalência de anemia nas mães $(21,8 \%)$ e filhos menores de 36 meses (57,6\%), a diferença percentual de 35,8\% para um erro de diferença (Ed\%) de 2,6, foi estatisticamente significante $(\mathrm{p}<0,01)$. Já na comparação entre mães e filhos com 36 meses e mais, a diferença percentual $(2,8 \%)$ equivale ao erro $(2,9 \%)$, não sendo, assim, estatisticamente significante.

Na Tabela 4 está descrita a relação entre as variáveis biológicas, socioeconômicas, ambientais e de acesso a serviços como fatores associados à anemia das mães e filhos. Nas mães, quatro fatores achavam-se estatisticamente relacionados ao risco de anemia: o regime de ocupação da residência (cessão/ 
Tabela 1 - Características amostrais (mães e filhos) segundo espaços geográficos do Estado de Pernambuco, 1997. Table 1 - Sample characteristics (mothers and children) according to geographical area in the state of Pernambuco.

\begin{tabular}{|c|c|c|c|c|c|c|c|c|c|}
\hline \multirow{2}{*}{ Variáveis analisadas } & & \multicolumn{2}{|c|}{ Estado } & \multicolumn{2}{|c|}{$\mathrm{RMR}^{*}$} & \multicolumn{2}{|c|}{ IU* } & \multicolumn{2}{|c|}{$I R^{*}$} \\
\hline & & No & $\%$ & $\mathrm{~N}^{\circ}$ & $\%$ & No & $\%$ & $\mathrm{~N}^{\circ}$ & $\%$ \\
\hline \multirow{3}{*}{ Idade da mãe } & $<20$ anos & 79 & 9,8 & 32 & 11,3 & 27 & 10,0 & 20 & 7,9 \\
\hline & $20 \mid-30$ anos & 450 & 55,8 & 165 & 58,3 & 159 & 58,7 & 126 & 50,0 \\
\hline & $\geq 30$ anos & 277 & 34,4 & 86 & 30,4 & 85 & 31,3 & 106 & 42,1 \\
\hline \multirow{2}{*}{ Idade da criança } & $<36$ meses & 559 & 67,6 & 201 & 64,0 & 172 & 67,5 & 186 & 72,1 \\
\hline & $36 \mid-60$ meses & 268 & 32,4 & 113 & 36,0 & 83 & 32,5 & 72 & 27,9 \\
\hline \multirow{3}{*}{ Peso ao nascer } & $<2500 \mathrm{~g}$ & 63 & 7,9 & 33 & 10,8 & 15 & 6,2 & 15 & 6,8 \\
\hline & $2500 \mid-3000 \mathrm{~g}$ & 159 & 20,0 & 82 & 26,8 & 44 & 18,2 & 33 & 14,9 \\
\hline & $\geq 3000 \mathrm{~g}$ & 574 & 72,1 & 191 & 62,4 & 183 & 75,6 & 173 & 78,3 \\
\hline \multirow{4}{*}{ Anos de estudo das mães } & $0-4$ & 322 & 40,1 & 68 & 24,5 & 77 & 28,4 & 177 & 70,0 \\
\hline & $4 \mid-8$ & 272 & 33,9 & 107 & 38,5 & 101 & 37,3 & 64 & 25,3 \\
\hline & $8 \mid-12$ & 160 & 20,0 & 77 & 27,7 & 75 & 27,7 & 8 & 3,2 \\
\hline & $\geq 12$ & 48 & 6,0 & 26 & 9,3 & 18 & 6,6 & 4 & 1,5 \\
\hline \multirow{3}{*}{ Renda familiar per capita } & $<0,25 \mathrm{SM}$ & 276 & 33,7 & 86 & 27,7 & 62 & 24,5 & 128 & 50,0 \\
\hline & $0,25 \mid-0,50 \mathrm{SM}$ & 224 & 27,3 & 76 & 24,5 & 63 & 24,9 & 85 & 33,2 \\
\hline & $\geq 0,50 \mathrm{SM}$ & 319 & 39,0 & 148 & 47,8 & 128 & 50,6 & 43 & 16,8 \\
\hline \multirow{3}{*}{ No pessoas da família } & $<5$ pessoas & 293 & 36,3 & 94 & 33,2 & 112 & 41,3 & 87 & 34,4 \\
\hline & $5 \mid-10$ pessoas & 428 & 53,0 & 155 & 54,8 & 132 & 48,7 & 141 & 55,6 \\
\hline & $\geq 10$ pessoas & 86 & 10,7 & 34 & 12,0 & 27 & 10,0 & 25 & 10,0 \\
\hline \multirow{3}{*}{$\begin{array}{l}\text { Regime de ocupação da } \\
\text { residência }\end{array}$} & Própria & 493 & 61,1 & 184 & 65,0 & 161 & 59,4 & 148 & 58,5 \\
\hline & Alugada & 152 & 18,8 & 64 & 22,6 & 77 & 28,4 & 11 & 4,3 \\
\hline & Cedida ou invadida & 162 & 20,1 & 35 & 12,4 & 33 & 12,2 & 94 & 37,2 \\
\hline \multirow{2}{*}{ Esgotamento sanitário } & Com rede geral & 210 & 26,0 & 77 & 27,2 & 133 & 49,1 & 0 & 0 \\
\hline & Sem rede geral & 597 & 74,0 & 206 & 72,8 & 138 & 50,9 & 253 & 100,0 \\
\hline \multirow{2}{*}{ Destino do lixo } & Coletado & 484 & 60,0 & 209 & 73,9 & 220 & 81,2 & 55 & 21,7 \\
\hline & Não coletado & 323 & 40,0 & 74 & 26,1 & 51 & 18,8 & 198 & 78,3 \\
\hline \multirow{3}{*}{ Assistência pré-natal } & Não fez pré-natal & 156 & 19,3 & 31 & 11,0 & 28 & 10,3 & 97 & 38,3 \\
\hline & $1 \mid-4$ consultas & 168 & 20,8 & 43 & 15,2 & 54 & 20,0 & 71 & 28,1 \\
\hline & $\geq 4$ consultas & 483 & 59,9 & 209 & 73,8 & 189 & 69,7 & 85 & 33,6 \\
\hline \multirow{2}{*}{ Visita do agente de saúde } & Sim & 262 & 32,8 & 95 & 34,3 & 64 & 23,6 & 103 & 40,9 \\
\hline & Não & 538 & 67,2 & 182 & 65,7 & 207 & 76,4 & 149 & 59,1 \\
\hline \multirow{3}{*}{$\begin{array}{l}\text { Distância do serviço de } \\
\text { saúde }\end{array}$} & $<1 \mathrm{~km}$ & 415 & 54,2 & 131 & 51,4 & 218 & 81,0 & 66 & 27,4 \\
\hline & $1 \mid-3 \mathrm{~km}$ & 155 & 20,3 & 100 & 39,2 & 39 & 14,5 & 16 & 6,6 \\
\hline & $\geq 3 \mathrm{~km}$ & 195 & 25,5 & 24 & 9,4 & 12 & 4,5 & 159 & 66,0 \\
\hline
\end{tabular}

*RMR: Região Metropolitana do Recife; * IU: Interior Urbano;*IR: Interior Rural; SM: salário mínimo.

${ }^{*}$ RMR: Recife Metropolitan Region; *UI:Urban Interior; RI Rural Interior; SM: minimal wage

invasão), a falta de tratamento do lixo, a falta de assistência pré-natal e a distância do serviço de saúde. Estas condições eram também, fatores de risco para a anemia dos filhos. No entanto, em relação às crianças, mais sete outras variáveis achavam-se associadas à ocorrência do problema: a idade da mãe, a idade da criança, o espaço geográfico (meio rural), a baixa escolaridade da mãe, a renda familiar abaixo de 0,5 salários mínimo, o maior número de membros da família e a falta de esgotamento sanitário ligado à rede geral de esgoto.

\section{Discussão}

O estudo aqui apresentado resgata um aspecto até então não explorado nas avaliações epidemiológicas das anemias: a prevalência e os fatores associados à dis- 
Tabela 2 - Prevalência de anemia em mães e filhos referentes à amostra total (A) e ao pareamento biológico (B), por estratos geográficos em Pernambuco, 1997.

Table 2 - Prevalence of anemia in mothers and children in the overall sample $(A)$ and biological pairing (B) per geographic strata in Pernambuco, Brazil, 1997.

\begin{tabular}{|c|c|c|c|c|c|c|c|c|c|}
\hline \multirow{2}{*}{\multicolumn{2}{|c|}{$\begin{array}{l}\text { Características dos grupos } \\
\text { estudados }\end{array}$}} & \multicolumn{2}{|c|}{ Estado } & \multicolumn{2}{|c|}{ RMR } & \multicolumn{2}{|c|}{ IU } & \multicolumn{2}{|c|}{ IR } \\
\hline & & No & $\%$ & No & $\%$ & No & $\%$ & No & $\%$ \\
\hline \multicolumn{10}{|c|}{ (A) Conjunto amostral (total) } \\
\hline & Anêmica & 176 & 21,8 & 70 & 24,7 & 43 & 15,9 & 63 & 24,9 \\
\hline \multirow[t]{3}{*}{ Mães } & Não anêmica & 631 & 78,2 & 213 & 75,3 & 228 & 84,1 & 190 & 75,1 \\
\hline & Total da amostra & 807 & 100,0 & 283 & 100,0 & 271 & 100,0 & 253 & 100,0 \\
\hline & Anêmico & 388 & 46,9 & 137 & 43,6 & 105 & 41,2 & 146 & 56,6 \\
\hline \multirow[t]{2}{*}{ Filhos } & Não anêmico & 439 & 53,1 & 177 & 56,4 & 150 & 58,8 & 112 & 43,4 \\
\hline & Total da amostra & 827 & 100,0 & 314 & 100,0 & 255 & 100,0 & 258 & 100,0 \\
\hline \multicolumn{10}{|c|}{ (B) Pareamento biológico } \\
\hline & Anêmica & 113 & 21,6 & 36 & 24,7 & 34 & 18,4 & 43 & 22,4 \\
\hline \multirow[t]{3}{*}{ Mães } & Não anêmica & 410 & 78,4 & 110 & 75,3 & 151 & 81,6 & 149 & 77,6 \\
\hline & Total da amostra & 523 & 100,0 & 146 & 100,0 & 185 & 100,0 & 192 & 100,0 \\
\hline & Anêmico & 249 & 46,6 & 66 & 42,9 & 74 & 41,6 & 109 & 54,0 \\
\hline \multirow[t]{2}{*}{ Filhos } & Não anêmico & 285 & 53,4 & 88 & 57,1 & 104 & 58,4 & 93 & 46,0 \\
\hline & Total da amostra & 534 & 100,0 & 154 & 100,0 & 178 & 100,0 & 202 & 100,0 \\
\hline
\end{tabular}

Tabela 3 - Relação entre a condição das mães (anêmicas x normais) e dos respectivos filhos, segundo espaços geográficos, no estado de Pernambuco (1997)

Table 3 - Relationship between status of mothers (anemic and normal) and their respective children, according to geographical area in the state of Pernambuco.

\begin{tabular}{|c|c|c|c|c|c|c|c|}
\hline \multirow{3}{*}{$\begin{array}{l}\text { Estrato } \\
\text { geográfico }\end{array}$} & \multirow{3}{*}{$\begin{array}{l}\text { Condição } \\
\text { das mães }\end{array}$} & \multicolumn{4}{|c|}{ Condição dos filhos } & \multicolumn{2}{|c|}{ Estatística } \\
\hline & & \multicolumn{2}{|c|}{ Anêmicos } & \multicolumn{2}{|c|}{ Normais } & \multirow{2}{*}{ RP (IC: 95\%) } & \multirow{2}{*}{ Valor $p^{*}$} \\
\hline & & No & $(\%)$ & No & (\%) & & \\
\hline \multirow[t]{2}{*}{ Estado } & Anêmicas & 63 & 66,3 & 32 & 33,7 & $1,45(1,21-1,74)$ & \multirow{2}{*}{$<0,001$} \\
\hline & Normais & 160 & 45,7 & 190 & 54,3 & 1,00 & \\
\hline \multirow[t]{2}{*}{ RMR } & Anêmicas & 21 & 67,7 & 10 & 32,3 & $1,62(1,14-2,29)$ & \multirow{2}{*}{0,013} \\
\hline & Normais & 36 & 41,9 & 50 & 58,1 & 1,00 & \\
\hline \multirow[t]{2}{*}{ I.U. } & Anêmicas & 16 & 55,2 & 13 & 44,8 & $1,34(0,91-1,98)$ & \multirow{2}{*}{0,168} \\
\hline & Normais & 53 & 41,1 & 76 & 58,9 & 1,00 & \\
\hline \multirow[t]{2}{*}{ I.R. } & Anêmicas & 26 & 74,3 & 9 & 25,7 & $1,41(1,10-1,82)$ & \multirow{2}{*}{0,021} \\
\hline & Normais & 71 & 52,6 & 64 & 47,4 & 1,00 & \\
\hline
\end{tabular}

*Qui-Quadrado de Pearson / *Pearson's Chi-square test

tribuição das anemias no binômio mãe/ filho, em estudo de base populacional. No caso da desnutrição energético-protéica (DEP) e sobrepeso/obesidade, há um ensaio com este enfoque analisando a relação do problema entre as crianças e suas mães ${ }^{13}$. Nosso estudo, além de constituir uma possível situação basal sobre as anemias na segunda metade da década passada, pode despertar o interesse para uma linha própria de pesquisas, ou seja, o comportamento das anemias e outros problemas carenciais a partir do micro-ambiente familiar.

Três condições importantes restringem as informações disponíveis sobre as anemias em nosso país: a limitada abrangência terri- 
Tabela 4 - Fatores associados à ocorrência da anemia em mães e filhos, no estado de Pernambuco (1997)

Table 4 - Factors associated with the occurrence of anemia in mothers and children, in the state of Pernambuco.

\begin{tabular}{|c|c|c|c|c|}
\hline \multirow{2}{*}{ Variáveis analisadas } & \multirow{2}{*}{$\begin{array}{c}\text { Mães } \\
\text { RP (IC 95\%) }\end{array}$} & \multicolumn{3}{|c|}{ Filhos } \\
\hline & & $\mathrm{p}$ & $\mathrm{RP}(\mathrm{IC} 95 \%)$ & $\mathrm{p}$ \\
\hline \multicolumn{5}{|l|}{ Biológicas } \\
\hline Idade da mãe ( $<20$ anos)/ 20-30 anos & $1,36(0,91-2,03)$ & 0,140 & $1,30(1,04-1,63)$ & 0,038 \\
\hline Idade da criança $(<3$ anos)/ 3-6 anos & * & & $2,34(1,87-2,92)$ & $<0,001$ \\
\hline Peso ao nascer $(<2.500 \mathrm{~g}) / \geq 3.000 \mathrm{~g}$ & $*$ & & $1,25(0,99-1,57)$ & 0,090 \\
\hline Espaço geográfico (rural)/ Região Metropolitana do Recife & $1,01(0,75-1,35)$ & 0,964 & $1,30(1,10-1,53)$ & 0,002 \\
\hline \multicolumn{5}{|l|}{ Condições sócio-econômicas } \\
\hline Anos de estudo da mãe $(<4$ anos $) / \geq 12$ anos & $1,05(0,60-1,83)$ & 0,860 & $1,96(1,08-3,57)$ & 0,006 \\
\hline Renda familiar per capita: $(<0,5) \mathrm{SM} / \geq 0,50 \mathrm{SM}$ & $1,13(0,86-1,48)$ & 0,372 & $1,37(1,16-1,61)$ & $<0,001$ \\
\hline$N^{\circ}$ de pessoas da família $(<5) / \geq 5$ & $0,91(0,69-1,18)$ & 0,467 & $1,18(1,00-1,39)$ & 0,041 \\
\hline Regime de ocupação da residência (cessão/invasão)/própria & $1,74(1,32-2,31)$ & 0,001 & $1,29(1,10-1,52)$ & 0,003 \\
\hline \multicolumn{5}{|l|}{ Condições de saneamento } \\
\hline Esgotamento sanitário (com rede geral) / sem rede geral & $1,24(0,90-1,70)$ & 0,186 & $1,51(1,23-1,86)$ & $<0,001$ \\
\hline Destino do lixo (não coletado)/ coletado & $1,37(1,05-1,77)$ & 0,018 & $1,33(1,15-1,54)$ & $<0,001$ \\
\hline \multicolumn{5}{|l|}{ Acesso aos serviços de saúde } \\
\hline Assistência pré-natal (sem assistência)/ 4 e + consultas & $1,38(1,01-1,90)$ & 0,048 & $1,32(1,12-1,56)$ & 0,002 \\
\hline Visita do agente de saúde & $1,12(0,84-1,49)$ & 0,432 & $1,01(0,87-1,18)$ & 0,889 \\
\hline Distância do serviço de saúde $(>3 \mathrm{~km}) /<1 \mathrm{~km}$ & $1,38(1,02-1,86)$ & 0,039 & $1,28(1,08-1,51)$ & 0,006 \\
\hline
\end{tabular}

* A hipótese não seria pertinente $/{ }^{*}$ Not pertinent hypothesis

${ }^{* *}$ Qui-quadrado de Pearson /**Pearson's Chi-square test

( / ) Razão de prevalência de Mantel Haenzel entre dois grupos, sendo o segundo o grupo de referência.

(/) Mantel Haenzel prevalence ratio between two groups, the second of which is the reference group.

torial dos estudos disponíveis; o fato de que, no período reprodutivo da mulher, a maior parte dos levantamentos se referem apenas a grupos de gestantes atendidas em serviços de saúde de áreas urbanas; e a raridade dos estudos analíticos, abordando os fatores relacionados com a ocorrência do problema. Poder-se-ia, acrescentar um quarto aspecto de interesse simultaneamente descritivo, analítico e programático: a abordagem das anemias sob a ótica do aglomerado familiar que, afinal de contas, representa o próprio micro-ambiente onde ocorre o processo saúde/doença ${ }^{14}$. É uma peculiaridade singularmente relevante para a compreensão do problema alimentar/nutricional, além de representar uma das abordagens assumidas na concepção e nas operações do Programa de Saúde da Família, no Brasil e em vários países no mundo.

Com referência à anemia nas mães, a situação não pode ser analisada comparativamente com qualquer outra unidade federativa do país, devido à inexistência de estudos de base populacional sobre o problema em outros Estados da federação, com exceção do Piauí. Neste Estado, 26,2\% das mães não gestantes apresentavam anemia ${ }^{9}$. Em São Leopoldo, RS, em estudo recente, detectou-se prevalência de 19,2\% de anemia, em mulheres não gestantes de 20 a 60 anos $^{15}$. As poucas avaliações disponíveis no Brasil se aplicam a mulheres grávidas atendidas em exames pré-natais e em espaços geográficos restritos, limitandose à cobertura operacional dos respectivos serviços de saúde ${ }^{10,16}$.

Santos ${ }^{10}$ estima que, em Pernambuco (2002), a prevalência de anemia em mulheres grávidas seria em torno de $30 \%$, o que se distancia do resultado de $21,8 \%$ aqui relatado. Pelo critério do Comitê WHO/ UNICEF $^{2}$ seria uma prevalência moderada. Outra pesquisa realizada na área urbana de Ribeirão, Zona da Mata de Pernambuco, evidenciou uma prevalência de $16,2 \%{ }^{17}$.

No Nordeste brasileiro e em outras áreas do país como um todo, são descritas 
freqüências bem mais elevadas de anemia em gestantes, com um intervalo modal de ocorrência de $30 \%$ a $40 \%$ das observações ${ }^{18}$. É bem possível que, no caso das gestantes, a anemia não represente uma comorbidade específica da gravidez, mas o agravamento de uma condição carencial que precede à própria concepção.

No âmbito internacional, os resultados do presente estudo concordam com os dados relatados por Shamah-Levy et al. em quatro regiões do México, com $20,8 \%$ das mulheres não grávidas classificadas como anêmicas ${ }^{19}$. Segundo aWHO/UNICEF/UNU (2001), freqüências bem mais elevadas seriam encontradas na África (40\%), enquanto no Sul da Ásia alcançariam $74 \%{ }^{3}$.

No que se refere às crianças, os conhecimentos acumulados sobre a ocorrência de anemias no Estado de Pernambuco, no Nordeste e no país são bem mais consistentes, embora ainda limitados para conclusões mais seguras. Assim, é interessante assinalar que a prevalência de $46,7 \%$ para o total de menores de 5 anos se equivale aos resultados obtidos por Monteiro et al. na cidade de São Paulo (46,9\%), Assis et al. (46,3\%) em Salvador, e 47,8\% encontrado por Silva et al. em Porto Alegre, $\mathrm{RS}^{4,20,21}$. Estes valores acham-se acima das prevalências descritas por Oliveira et al. no Estado da Paraíba ${ }^{5}$ e, ainda, das ocorrências registradas nos Estados de Sergipe ${ }^{10}$ e Piauí ${ }^{9}$. Já em Pelotas, RS, um estudo realizado por Assunção et al. resultou em uma prevalência de anemia de $30,2 \%$ em menores de 6 anos $^{22}$.

A situação comparativa de mães e crianças difere significativamente, desde que a prevalência em menores de 5 anos, com exceção do IU, fosse mais de duas vezes superior àquela registrada nas mulheres para o nível estadual. De forma análoga, persiste a diferença significante quando se comparam as duplas mães/filhos por pareamento biológico. Por outro lado, constata-se que as mães anêmicas têm uma maior probabilidade (praticamente dois terços) de se associar a filhos anêmicos. No entanto, mesmo mães com níveis normais de hemoglobina apresentam uma chance elevada de ocorrência de anemia em seus filhos: $45,2 \%$ para todo o Estado e em torno de $41 \%$ para a RMR e o IU. As diferenças entre mães e filhos biológicos foram estatisticamente significantes, com exceção apenas do IU. Vale observar que os resultados de Pernambuco diferem das estatísticas consolidadas em nível internacional nas nações em desenvolvimento, nas quais as mulheres, gestantes ou não, apresentam maiores prevalências que as crianças menores de cinco anos ${ }^{3}$.

Para o conjunto dos resultados do Estado de Pernambuco, as diferenças entre mães e filhos desapareceram quando os resultados das crianças foram desagregados em dois estratos, acima ou abaixo da idade de três anos. De fato, a partir desta idade, a prevalência de anemia entre mães e filhos passa a ser estatisticamente equivalente. Isto levaria a duas observações imediatas: a diferença entre mães e filhos menores de cinco deve-se, efetivamente, à participação do grupo de crianças menores de três anos; $\mathrm{e}$ é muito provável que, depois de 36 meses de idade, a alimentação das crianças passaria a ser semelhante ao padrão alimentar da família ou, pelo menos, ao perfil alimentar das mães.

Um objetivo muito interessante do inventário analítico consiste em relacionar a distribuição das anemias em mães e filhos segundo os fatores mais comumente utilizados em estudos epidemiológicos sobre problemas nutricionais ${ }^{23}$. Assim, para as mães, foram identificados quatro fatores associados à ocorrência de anemia: o regime de ocupação da residência (cessão/invasão representando um handicap), falta de assistência pré-natal, falta de tratamento do lixo domiciliar e distância do serviço de saúde. Estas situações expressam condições de vida marcantemente adversas que podem contribuir, de forma isolada ou interativa, com a gênese das anemias. Fazem parte do contexto de pobreza, ainda que a renda familiar, isoladamente, não possibilite um efeito estatisticamente discriminante da freqüência de anemia nas mães. Ademais, esta singularidade justifica o juízo de não limitar a expressão da pobreza à visão 
reducionista da renda, recomendando-se analisar outras variáveis, particularmente o acesso a bens materiais, para compreender as manifestações do estrato social no processo saúde/doença e, sobretudo, na situação nutricional ${ }^{24,25}$. Esta consideração se particulariza ainda mais no caso das anemias, considerada a mais democrática das deficiências nutricionais, por se distribuir endemicamente em todos os estratos de renda ${ }^{26}$.

As variáveis correlacionadas com a anemia das mães também foram significantes em relação aos filhos. Em relação às crianças aparecem mais sete fatores de risco: a idade abaixo de três anos, a idade das mães $(<20$ anos), o baixo nível de escolaridade da mãe, o espaço geográfico, o número de pessoas da família, o esgotamento sanitário e a baixa renda familiar.

$\mathrm{O}$ fato de a freqüência de anemia nas crianças ser mais de duas vezes superior à encontrada nas mães, já se torna indicativo de que, além da magnitude do problema, as crianças podem incorporar fatores adicionais de risco. Isto se evidencia na constatação de que a idade abaixo de três anos representa uma clara diferenciação em relação às crianças maiores, quando as prevalências do problema passam a se equivaler às encontradas nas mães. Por outro lado, a adição de mais três variáveis de caráter socioeconômico (a baixa escolaridade das mães, a falta de esgotamento sanitário e a renda familiar), expressaria que, no caso de Pernambuco, em discordância com a situação mundial, as crianças seriam bem mais vulneráveis que suas mães às adversidades socioeconômicas, mesmo em relação a uma "endemia democrática" como a anemia.

Nos dois casos (mães e crianças) há uma informação relevante que não foi inventariada no estudo: o consumo alimentar. De acordo com as Nações Unidas (UNICEF/ UNU/WHO), a maior parte das anemias derivariam da carência primária de ferro alimentar e, particularmente, da baixa disponibilidade de ferro-heme ${ }^{3}$. No estado de Pernambuco, Osório et al. ${ }^{27}$ demonstraram que o nível de consumo de ferro heme se correlacionava com a idade das crianças, elevando-se de $0,3 \mathrm{mg} /$ dia na faixa de 6 - 11 meses, para 0,8 mg/dia, no grupo de 48-59 meses. Num outro trabalho ${ }^{28}$, demonstra-se o papel do leite de vaca como um fator de risco das anemias em crianças, tal como verificaram Levy-Costa e Monteiro ${ }^{29}$ na cidade de São Paulo. São informações importantes para explicar a maior prevalência de anemia em menores de três anos.

O trabalho tem evidentes limitações, a partir da circunstância de que não foi originariamente concebido e desenhado para um inquérito de campo específico sobre anemia em mães e crianças, derivando de um banco de dados em que os objetivos centrais eram outros. Não se conta, além da hemoglobina, com outros indicadores hematimétricos e bioquímicos, como ferritina, capacidade de ligação do ferro, protoporfirina eritrocitária e outros, para caracterizar a provável etiologia do problema. Não se dispõe de dados sobre o consumo alimentar e, por extensão, a quantidade de ferro biodisponível na dieta de mães e crianças, o que constitui por sinal, uma informação muito difícil de ser obtida e interpretada. No entanto, como se demonstra em seus resultados e questionamentos, representam subsídios básicos para o próprio aprofundamento de novos estudos. Seria interessante uma análise multivariada dos fatores associados às anemias de mães e filhos, o que enfrenta evidentes dificuldades, desde que a principal informação (o consumo alimentar de mães e filhos e seus respectivos valores nutricionais) não está disponibilizada; ademais, as crianças menores de 5 anos apresentam uma curva de distribuição de anemia bastante heterogênea, com máximo de prevalência em torno de 12-15 meses, decrescendo rapidamente a seguir; as mulheres em idade fértil têm necessidades de ferro bem diferenciadas das suas famílias e, portanto, em desacordo com as adequações de consumo que habitualmente são estimadas para o contexto familiar.

Conclusivamente, o estudo evidencia que as prevalências de anemia em mães e 
crianças diferem significativamente entre si e, em menor escala, nos espaços geográficos. As mães anêmicas apresentam um risco maior de ter filhos menores de 5 anos com o mesmo problema. Demonstrou-se que a anemia das mães se associa ao regime de ocupação atípica da residência, à falta de tratamento do lixo domiciliar, de assistência pré-natal e a distância dos serviços de saúde. As crianças incorporam mais sete condições de risco: sua idade (menor de três anos), a idade da mãe (menor de 20 anos), o espaço geográfico (meio rural), maior número de pessoas da família, a baixa escolaridade da mãe, a baixa renda familiar e a deficiência de esgotamento sanitário.

\section{Referências}

1. Demaeyer E, Dallman P, Gurney JM. Preventing and controlling iron deficiency anemia through primary healt care. Geneva: World Health Organization; 1989. (A guide for health administrations and programme managers).

2. United Nations Children's Fund, World Health Organization. Prevention and control of iron deficiency anaemia in women and children. Geneve: UNICEF/WHO; 1999.

3. World Health Organization, United Nations Children's Fund, United Nations University. Iron deficiency anaemia: assessment, prevention and control. A guide for programme managers. Geneve: WHO/UNICEF/UNU; 2001.

4. Monteiro CA, Szarfarc SC, Mondini L. Tendência secular da anemia na infância na cidade de São Paulo (1984-1996). Rev Saúde Pública 2000; 34 (S6): 62-72.

5. Oliveira RS et al. Magnitude, distribuição espacial e tendência da anemia em pré-escolares da Paraíba. Rev Saúde Pública 2002; 36(1): 26-32.

6. Veras ICL. Aspectos alimentares e nutricionais de escolares em educandários públicos do bairro da Várzea, Recife-PE. [tese de doutorado]. Recife: Departamento de Nutrição da UFPE; 2004

7. West KP, Jr. Extent of vitamin A deficiency among preschool children and women of reproductive age. J Nutr 2002; 132 : S2857-66.

8. Batista Filho M, Romani SAM. (orgs). Alimentação, Nutrição e Saúde no Estado de Pernambuco. Recife; Instituto Materno Infantil de Pernambuco (IMIP). Série Publicações Cientificas do Instituto Materno Infantil de Pernambuco (IMIP) n.7; 2002.

9. Governo do Estado do Piauí, UFPI, UNICEF. Crianças e adolescentes no Piaui - Saúde, Educação e Trabalho. Governo do Estado do Piauí, Teresina; 1992.

10. Santos LMP. (org.). Bibliografia sobre deficiência de micronutrientes no Brasil: 1990-2000. Brasília: OPAS (Organização Panamericana da Saúde), v.2- Anemia; 2002.
11. Lima e Silva SC, Batista Filho, M. Anemia em mães e filhos no Estado de Pernambuco. Série Publicações Científicas do Instituto Materno Infantil Prof. Fernando Figueira n 9; 2005.

12. National Center For Health Statistics (NCHS). Growth curves for children birth - 18 years. Washington, D.C.: Governamment Print Office; 1977. (Vital Health Statistics Series, v.11, n.165).

13. Engstron EM, Anjos LA. Relação entre o estado nutricional materno e sobrepeso nas crianças brasileiras. Rev Saúde Pública 1996; 30(3): 233-9.

14. Ministério da Saúde. Saúde da Familia no Brasil: uma análise de indicadores selecionados: 1998-2004. Brasília: Ministério da Saúde; 2006.

15. Fabian C, Olinto MTA, Costa JSD, Nácul LC. Prevalência de anemia e fatores associados em mulheres adultas residentes em São Leopoldo, Rio Grande do Sul, Brasil. Cad Saúde Pública 2007; 23(5):1199-205.

16. Arruda IKG. Deficiencia de ferro, de folato e anemia em gestantes atendidas no Instituto Materno Infantil de Pernambuco: magnitude, fatores de riscos e algumas implicações nos seus conceptos. [tese de doutorado]. Recife: Departamento de Nutrição da UFPE; 1997.

17. Ferreira LOC, Batista Filho M. Um modelo para avaliação rápida da situação nutricional e de saúde de crianças e mães no "Dia Nacional da Vacinação". Rev Bras Saúde Materno Infantil 2001; 1(2): 145-54.

18. Batista Filho M. O controle das anemias no Brasil. Rev Bras Saúde Materno Infantil 2004; 4(2): 121-23.

19. Shamah-Levy T, Villalpando S, Rivera JA, Mejía-Rodríguez F, Camacho-Cisneros M, Monterrubio E. A. Anemia in Mexican women: a public health problem. Salud Pública de México 2003; 45 (S4): 499-507.

20. Assis AMO et al. Distribuição da anemia em pré-escolares do semi-árido da Bahia. Cad Saúde Pública 1997; 13(2): 237-43. 
21. Silva LSM, Giugliani ERJ, Aerts DRGC. Prevalência e determinantes de anemia em crianças de Porto Alegre, RS, Brasil. Rev Saúde Pública 2001; 35(1): 66-73.

22. Assunção MCF, Santos IS, Barros AJD, Gigante, DP, Victora, CG. Anemia em menores de seis anos: estudo de base populacional em Pelotas, RS. Rev Saúde Pública 2007; 41(3): 328-35.

23. Batista Filho M, Rissin A. A transição nutricional no Brasil: tendências regionais e temporais. Cad Saúde Pública 2003; 19 (S1): 181-91.

24. Rissin A. Desnutrição em crianças menores de cinco anos no Estado de Pernambuco: uma análise de relações causais hierarquizadas. Recife: Editora. Liceu; 2003.

25. Sousa FJPS. Pobreza, desnutrição e mortalidade infantil: condicionantes socioeconômicos. Fortaleza: UNICEF; 1992.
26. Szarfarc SC. Diagnóstico da deficiência de ferro na infância. Rev Saúde Pública 1985; 19: 278-84.

27. Osório MM, Lira PIC, Ashmorth A. Consumo e biodisponibilidade de ferro das crianças de 6-59 meses de idade em Pernambuco. In: Batista Filho M, Romami SAM. (Org.) Alimentação, Nutrição e Saúde no Estado de Pernambuco: espacialização e fatores sócio-econômicos. Série de Publicações Científicas do Instituto Materno-Infantil de Pernambuco, n.7. Recife; 2002.

28. Oliveira MAA, Osório MM, Raposo MCF. Concentração de hemoglobina e anemia em crianças no Estado de Pernambuco, Brasil: fatores sócio-econômicos e de consumo alimentar associados. Cad Saúde Pública 2006; 22(10): 2169-78.

29. Levy-Costa RB, Monteiro CA. Consumo de leite de vaca e anemia na infância no município de São Paulo. Rev Saúde Pública 2004; 38: 797-803.

Recebido em: 27/02/07

Versão final reapresentada em: 04/03/08

Aprovado em: 19/03/08 